\title{
DESAFIOS E AVANÇOS PARA A ESTRUTURAÇÃO DE UMA COMUNIDADE DE PRÁTICA DE VISITAÇÃO EM ÁREAS PROTEGIDAS
}

\author{
Douglas de Souza Pimentel ${ }^{1}$ \\ Marcelo Derzi Vidal ${ }^{2}$ \\ Susy Rodrigues Simonetti ${ }^{3}$ \\ Camila Gonçalves de Oliveira Rodrigues ${ }^{4}$ \\ Sônia Maria Sfair Kinker ${ }^{5}$ \\ Alba Valéria Santos Simon ${ }^{6}$ \\ Eloise Silveira Botelho ${ }^{7}$
}

\section{RESUMO}

A Comunidade de Prática é formada por um grupo de pessoas com interesses comuns em um determinado assunto, que se reúnem periodicamente e estão conectados por algum tipo de comunicação virtual, para compartilhar conhecimento e aplicá-lo, estabelecer parcerias, desenvolver projetos, entre outras iniciativas. Nesse caso, o interesse comum é a visitação em áreas protegidas. O College of Forestry and Conservation of the University of Montana instituiu, desde 2013, uma parceria com o Instituto Chico Mendes de Conservação da Biodiversidade (ICMBio), por meio do Serviço Florestal dos Estados Unidos (USFS) e da United States Agency for International Development (USAID), com o objetivo de fomentar a criação de uma rede comunidade de prática - para educação, treinamento e pesquisa destinada a apoiar o manejo de áreas protegidas no Brasil. A parceria foi consolidada entre universidades brasileiras e dos Estados Unidos da América (EUA), e agências de gestão de áreas protegidas brasileiras. Definiu-

\footnotetext{
${ }^{1}$ Professor Associado da Universidade do Estado do Rio de Janeiro e da Universidade Federal Fluminense. E-mail: douglasgeia@gmail.com

${ }^{2}$ Analista Ambiental. Centro Nacional de Pesquisa e Conservação da Sociobiodiversidade Associada a Povos e Comunidades Tradicionais - CNPT/ICMBio. Doutor em Biodiversidade e Conservação.

E-mail: marcelo.vidal@icmbio.gov.br

${ }^{3}$ Professora adjunta do Curso de Bacharelado em Turismo e do Mestrado Interdisciplinar em Ciências Humanas PPGICH, ambos da Universidade do Estado do Amazonas - UEA e do Mestrado Profissional em Gestão de Áreas Protegidas - MPGAP/INPA. E-mail: $\underline{\text { ssimonetti@uea.edu.br }}$

${ }^{4}$ Professora Associada. Departamento de Administração e Turismo. Programa de Pós-Graduação em Práticas em Desenvolvimento Sustentável. Universidade Federal Rural do Rio de Janeiro. Doutora em Política e Gestão Ambiental. E-mail: camila.rodrigues.ufrrj@gmail.com

${ }^{5}$ Analista Ambiental. Parque Nacional da Tijuca - Instituto Chico Mendes de Conservação da Biodiversidade (PNT/ICMBio). Doutoranda em Sociedade e Conservação pela Universidade de Montana/EUA. E-mail: sonia.kinker@icmbio.gov.br

${ }^{6}$ Professora visitante do Programa de Pós Graduação em Ecoturismo e Conservação da Universidade Federal do Estado do Rio de Janeiro. E-mail: albasimon7@gmail.com

${ }^{7}$ Doutora em Ciências pelo Programa de Pós-graduação em Engenharia de Produção (COPPE/UFRJ). Professora Adjunta do Departamento de Turismo. e Patrimônio. Programa de Pós-Graduação em Ecoturismo e Conservação. Universidade Federal do Estado do Rio de Janeiro. E-mail: eloise.botelho@ unirio.br.
} 
se que o objetivo primordial dessa comunidade de prática seria a colaboração entre pesquisadores e gestores de Unidades de Conservação (UC) para o desenvolvimento de pesquisas sobre a visitação nessas áreas, além de estimular a utilização de dados e informações gerados pela parceria como subsídio para o planejamento e o manejo da visitação. O objetivo deste trabalho é apresentar uma análise dos encontros desta rede, sistematizando os desafios enfrentados na estruturação e funcionamento do que se denominou Encontro da Comunidade de Prática de Visitação em Áreas Protegidas (ECPVAP). A metodologia envolveu a análise de conteúdo dos relatórios disponíveis dos Encontros. Dentre os desafios elencados nos encontros, destaca-se o estabelecimento de estratégias para uma maior conexão, envolvimento, comunicação e relacionamento entre os visitantes e habitantes das áreas de abrangência das UC. No que diz respeito às linhas de atuação da comunidade, o tema referente ao "manejo" foi a que apresentou uma maior diversidade de tópicos que devem ser trabalhados. Ficou evidente que os objetivos e a função da comunidade estão bem definidos. Contudo, os membros do grupo continuam refletindo e buscando caminhos para superar alguns desafios para executar o planejamento das ações. Com relação ao perfil da comunidade, identificou-se que há maior proporção de participantes da região Sudeste, indicando a necessidade de se ampliar a participação de pesquisadores e gestores de outras regiões do país. Constatou-se que a participação de gestores aumentou entre o primeiro e terceiro encontros, mas diminuiu nos dois encontros seguintes. Quando houve maior presença de gestores, questões sobre o manejo foram o foco. Por fim, destaca-se que dentre os temas elencados para atuação conjunta entre gestores e pesquisadores, não houve aprofundamento sobre os temas da educação e interpretação ambiental.

Palavras Chave: Conservação ambiental; Manejo; Uso público

\section{CHALLENGES AND ADVANCES IN STRUCTURING A COMMUNITY OF PRACTICE ON VISITATION IN PROTECTED AREAS}

\section{ABSTRACT}

The Community of Practice is composed by a group of people with common interests in a particular subject, who meet periodically and are connected by some kind of virtual communication, to share knowledge and apply it, to establish partnerships, to develop projects, among other initiatives. In this case, the common interest is visitation in protected areas. Since 2013, the College of Forestry and Conservation of the University of Montana has established a partnership with the Instituto Chico Mendes de Conservação da Biodiversidade (ICMBio) through the United States Forest Service (USFS) and the United States Agency for International Development (USAID), with the objective of fostering the creation of a network - community of practice - for education, training, and research to support the management of protected areas in Brazil. The partnership was consolidated between Brazilian and United States (US) universities, and Brazilian protected areas management agencies. It was defined that the primary objective of this community of practice would be the collaboration between researchers and managers to develop research on visitation in protected areas, and to stimulate the use of data and information generated by the partnership as a subsidy for planning and management of public use. The objective of this paper is to present an analysis of the meetings of this network, systematizing the challenges faced in the structuring and functioning of what was called the Community Meeting of 
Visitation Practice in Protected Areas (ECPVAP). The methodology involved the content analysis of the available reports of the Meetings. Among the challenges listed in the meetings, we highlight the establishment of strategies for greater connection, involvement, communication and relationship between visitors and inhabitants of the areas protected areas. With regard to the community's lines of action, the "environmental management" theme presented the greatest diversity of topics that should be addressed. It was evident that the goals and function of the community are well defined. However, group members continue to reflect and look for ways to overcome some challenges in carrying out action planning. Regarding the profile of the community, it was identified that there is a higher proportion of participants from the Southeast region, indicating the need to increase the participation of researchers and managers from other regions of the country. It was found that the participation of managers increased between the first and third meetings, but decreased in the following two meetings. When there was a greater presence of managers, management issues were the focus. Finally, it is noteworthy that among the topics listed for joint action between managers and researchers, there was no deepening on the themes of education and environmental interpretation.

Key words: Environmental Conservation, Management, Public Use

\section{INTRODUÇÃO}

O conceito de Comunidade de Prática foi proposto pelo antropólogo Jean Lave e pelo educador Etienne Wenger na década de 1990, no livro "Situated Learning" (LAVE, WENGER, 1991). Posteriormente, Wenger expandiu significativamente o conceito em seu livro de 1998, "Community of Practice". Dessa maneira, há o entendimento que as relações estabelecidas em uma comunidade de prática são fluidas e informais, em um ambiente de aprendizado colaborador, em que são partilhados sistemas de crenças, experiências e identidades em comum. Sendo assim, as interações constituídas entre os membros não só favorecem a reflexão e a ação, em processo permanente e mútuo de reflexão e aprendizado como, também, propiciam a inovação ativada pelo repertório coletivo de experiências (SOUZA-SILVA, DAVEL, 2007).

Nessa mesma direção, Ipiranga, Faria e Amorim (2008) dão destaque ao processo de aprendizado, afirmando que as comunidades de prática possibilitam a aprendizagem a partir da vivência cotidiana, do trabalho e das práticas sociais, para além da perspectiva centrada apenas em métodos cognitivos. De fato, esse é um dos principais desafios à incorporação dos resultados obtidos a partir dos processos de aprendizado e inovação das comunidades de práticas nas suas instituições de origem. Isso não significa necessariamente, que as comunidades de prática devem se formalizar, ao contrário, seu aspecto informal e fluido é fundamental para o contínuo processo de aprendizagem e inovação. Disso, reside o paradoxo de uma comunidade de prática e um desafio a ser superado (WENGER, SNYDER, 2000).

Baseando nisso, o termo comunidade de prática é entendido nesse artigo como um grupo de pessoas com interesses comuns em um determinado assunto, que se reúne periodicamente para compartilhar conhecimento e aplicá-lo, estabelecer parcerias para um aprendizado coletivo e 
cooperativo. É através do processo de compartilhamento de informações e experiências com o grupo que os membros, sustentados pelo engajamento voluntário e mútuo, aprendem um do outro e têm a oportunidade de se desenvolver pessoal e profissionalmente (LAVE, WENGER, 1991). Dessa maneira, uma comunidade de prática pode evoluir naturalmente devido ao interesse comum dos membros em um domínio ou área particular, ou pode ser criada deliberadamente com o objetivo de obter conhecimento relacionado a um campo específico. No presente caso, o foco compartilhado é a visitação em áreas protegidas.

A Comunidade de Prática de Visitação em Áreas Protegidas (CPVAP) conta com o apoio de uma universidade americana, a Universidade de Montana (UM), e o Serviço Florestal dos Estados Unidos, que já trabalhavam em cooperação no Brasil. A UM já havia participado da criação e desenvolvimento de uma comunidade de prática que envolvia países do continente africano (África do Sul, Zambia, Botswana, Zimbabwe), que iniciou seus trabalhos se debruçando sobre o mesmo tema: visitação em áreas protegidas. Com essa experiência acumulada, a UM propôs às instituições gestoras de áreas protegidas no Brasil e às universidades brasileiras potencializar suas parcerias por meio da Comunidade.

O objetivo da CVAP é, além de aproximar pesquisadores e gestores de unidades de conservação com interesse na visitação em áreas protegidas, elaborar uma agenda de pesquisas de interesse comum e aplicar o resultado dessas pesquisas no manejo das unidades de conservação. Os encontros da comunidade são itinerantes (ocorre nas várias regiões do Brasil) e periódicos (uma vez a cada ano), com o intuito de identificar tendências e demandas locais, e para o estabelecimento de novas parcerias por meio do conhecimento do trabalho dos diversos pesquisadores e gestores. Para além dos encontros anuais, o grupo trabalha em várias frentes, tais como publicações conjuntas, participação em eventos e recentemente, parceria no desenvolvimento de projeto de fomento de iniciativas de turismo de base comunitária em uma Área de Proteção Ambiental (APA) Federal, além de outros.

Em março de 2015, foram convidadas universidades brasileiras e americanas, além dos órgãos gestores de UC para participar, de forma voluntária, do I Encontro CPVAP, realizado no Parque Nacional de Brasília (DF). Neste encontro, definiu-se que o objetivo primordial dessa comunidade de prática seria:

Promover e fortalecer a colaboração entre pesquisadores e gestores de Unidades de Conservação (UC) para o desenvolvimento de pesquisas sobre a visitação em áreas protegidas, além de estimular a utilização de dados e informações geradas pela pesquisa como subsídio para o planejamento e o manejo da visitação (COMUNIDADE DE PRÁTICA DE VISITAÇÃO EM ÁREAS PROTEGIDAS, 2015, p.3)

Esse objetivo balizou os encontros subsequentes, que ocorrem anualmente em diferentes regiões: o II Encontro da CPVAP, realizado em 2016, aconteceu na Universidade de Brasília, (Brasília - DF); a terceira edição no Parque Nacional da Serra dos Órgãos, na sede de Teresópolis (RJ), em 2017; o IV Encontro ocorreu na Universidade Estadual do Amazonas, em Manaus 
(AM), em 2018. Em 2019, o V Encontro da CPVAP aconteceu na Universidade Federal do Estado do Rio de Janeiro (UNIRIO), na cidade do Rio de Janeiro (COLLEGE OF FOREST AND CONSERVATION, 2015; COMUNIDADE DE PRÁTICA DE VISITAÇÃO EM ÁREAS PROTEGIDAS, 2017, 2018).

Os I e II Encontros buscaram a estruturação da CPVAP a partir do desenvolvimento de um plano de ação para os anos seguintes. A metodologia de trabalho ao longo dos dias de reunião baseou-se nas experiências individuais para a troca de experiências, expostas pelos participantes, pesquisadores e acadêmicos, que foram estimulados por questões orientadoras de forma a identificar o interesse científico e profissional de cada um dos participantes na pesquisa sobre visitação em áreas protegidas. Sendo assim, foram levantadas as perguntas de pesquisa sobre o tema da Comunidade de Prática; os desafios da academia em relação ao gerenciamento de uso público e áreas protegidas no Brasil; a identificação de lacunas existentes em relação ao tema e de que forma a CPVAP poderia ajudar a superar essas lacunas (COLLEGE OF FOREST AND CONSERVATION, 2015; COMUNIDADE DE PRÁTICA DE VISITAÇÃO EM ÁREAS PROTEGIDAS, 2017).

No III Encontro da CPVAP a metodologia de trabalho envolveu, novamente, a apresentação de cada participante e um resumo dos seus principais temas de pesquisa e atuação. Em seguida, diferentes tópicos foram debatidos, como as demandas de pesquisa apresentadas pelo ICMBio, pelo Instituto Estadual do Ambiente (INEA-RJ) e pelo Parque Nacional Serra dos Órgãos, que sediou o evento, além da troca de experiências sobre pesquisas/metodologias e as potencialidades e desafios para o desenvolvimento de novas pesquisas (COMUNIDADE DE PRÁTICA DE VISITAÇÃO EM ÁREAS PROTEGIDAS, 2017).

No IV Encontro, realizado em Manaus, foram desenvolvidos diferentes painéis relacionados às pesquisas sobre visitação nas UC da Amazônia e seus desafios e potencialidades. Realizou-se também uma oficina para a construção colaborativa de um roteiro para identificar o perfil e o gasto médio dos visitantes de UC, considerando também sugestões sobre métodos para amostragem, coleta e tratamento dos dados (COMUNIDADE DE PRÁTICA DE VISITAÇÃO EM ÁREAS PROTEGIDAS, 2018).

Na sequência, para facilitar a definição do escopo da CPVAP e o planejamento de suas ações foi realizada uma oficina que utilizou como inspiração a metodologia de Pesquisa Orientada para Ação e Decisão - PAD (MUTISSE, FONTOURA, QUIVE, 2019). Por meio da PAD, os participantes revisitaram os objetivos da Comunidade de Prática e classificaram por temas, as ações identificadas para alcançar os objetivos, estabelecendo um plano de trabalho realista para todos os envolvidos.

Na realização do V Encontro, ficou evidente que a Comunidade está estabelecida e que, de agora em diante, o trabalho não é mais de organização, mas de fortalecimento e consolidação das parcerias para promover e dar suporte à pesquisa e à gestão da visitação, podendo também ampliar o alcance da rede envolvendo um maior número de instituições. 
Diante desta contextualização sobre a Comunidade de Prática de Visitação em Áreas Protegidas no Brasil, o objetivo do presente artigo é sistematizar e discutir os desafios enfrentados e os avanços obtidos na sua estruturação e funcionamento, trazendo subsídios importantes para ações futuras em temas similares.

\section{METODOLOGIA}

Os temas debatidos durantes os encontros da CPVAP e os resultados obtidos foram registrados em relatórios técnicos que serviram de base para a análise apresentada neste trabalho. Dessa forma, para o alcance dos objetivos da pesquisa foi realizada uma análise dos três principais Relatórios (I, III e IV) da Comunidade de Prática de Visitação em Áreas Protegidas disponíveis, ou seja, o corpus documental constituiu-se dos relatórios de atividades dos Encontros de 2015, 2017 e 2018.

Os relatórios apontaram um caminho para a codificação do material; o que Bardin (1977) denomina de "Leitura Flutuante". Seguiu-se uma análise quantitativa dos dados de modo a sistematizar o número de participantes e diversidade de instituições representadas nos eventos e uma análise de conteúdo quali-quantitativa (BARDIN, 1977).

$\mathrm{Na}$ etapa seguinte, houve a determinação dos núcleos dos sentidos e unidades amostrais, que foram diferentes de acordo com a abordagem realizada a partir da análise de palavras e frases. A codificação dos documentos esteve centralizada nas palavras-chave do objetivo da CPVAP, bem como nos questionamentos, estes últimos classificados em nove núcleos de sentidos. A seguir, são apresentados os resultados da análise.

\section{RESULTADOS E DISCUSSÃO}

Considerando especificamente cada um dos três encontros analisados, pode-se observar que no primeiro evento, as linhas de atuação dos participantes estavam relacionadas aos temas: "Sociedade e Ambiente"; "Caracterização do visitante"; "Manejo"; e "Turismo e Estrutura", como apontado no Quadro 1.

No I Encontro, inicialmente, a comunidade elencou temas considerados importantes para a gestão da visitação em áreas protegidas. Estes foram agrupados em listas e enquadrados em três temas centrais: a promoção de conexões entre as pessoas e o ambiente; a relação do patrimônio natural com as experiências dos visitantes; e a construção/fortalecimento da resiliência de comunidades receptoras de turismo em áreas protegidas.

A partir dos temas centrais, foram identificados mais de 80 desafios para a gestão da visitação em áreas protegidas e, posteriormente, foram classificados como mais significativos os seguintes: as conexões entre sociedade e natureza como forma de assegurar o patrimônio natural para as experiências dos visitantes; a construção da resiliência da comunidade receptora; a relação limitada entre a sociedade e as áreas protegidas; a ausência de planejamento de uso público; a promoção do envolvimento da comunidade no planejamento e monitoramento do uso público; a capacitação para o manejo do uso público; a gestão da informação e a criação de uma 
estratégia de comunicação e marketing, bem como a implementação efetiva de programas e projetos de uso público. A figura a seguir, apresenta um quadro com as linhas de atuação dos participantes do I Encontro:

QUADRO 1- Apresenta as Linhas de atuação dos participantes do I Encontro da CPVAP

\begin{tabular}{|l|l|}
\hline \multicolumn{1}{|c|}{ Grupos } & \multicolumn{1}{c|}{ Linhas de atuação } \\
\hline Sociedade e Ambiente & $\begin{array}{l}\text { Interpretação ambiental; educação ambiental; conexão homem/natureza por } \\
\text { meio do fortalecimento da relação entre visitantes e UC, uso público e saúde } \\
\text { (qualidade de vida); voluntariado. }\end{array}$ \\
\hline Caracterização & Estimativa do número e perfil dos visitantes; capacidade de carga. \\
\hline Manejo & $\begin{array}{l}\text { Manejo de trilhas; monitoramento de impactos da visitação (econômicos, } \\
\text { sociais e ambientais); avaliação da efetividade de gestão do uso público; } \\
\text { ordenamento da visitação; impactos do uso público sobre a fauna. }\end{array}$ \\
\hline Turismo e estrutura & Concessões de serviços e parcerias privadas; turismo de base comunitária. \\
\hline
\end{tabular}

Fonte: Relatório do I Encontro da CPVAP, 2015.

No que se refere às principais demandas de pesquisa levantadas no III Encontro da CPVAP (2017), seis temas principais foram apontados, como demonstrado no Quadro 2. Neste quadro, pode-se destacar a questão da avaliação de impactos da visitação, com o maior número de tópicos para investigação.

QUADRO 2 - As principais demandas de pesquisa levantadas no III Encontro da CPVAP, agrupadas em seis categorias.

\begin{tabular}{|l|l|}
\hline \multicolumn{1}{|c|}{ Grupos } & \multicolumn{1}{|c|}{ Principais demandas de pesquisa e potenciais áreas para colaboração } \\
\hline Sociedade e \\
Ambiente & $\begin{array}{l}\text { Avaliação de Programas de IA e EA; ampliação do conhecimento sobre temas } \\
\text { (geodiversidade, sociodiversidade e biodiversidade); contexto histórico de trilhas; } \\
\text { História institucional do parque; Inclusão e aproximação com as comunidades; } \\
\text { relação da gestão da UC com as comunidades, benefícios econômicos; perfil de } \\
\text { gastos dos visitantes no entorno e na UC; associar impactos econômicos à } \\
\text { benefícios sociais; como transformar o visitante num aliado da conservação }\end{array}$ \\
\hline Turismo & $\begin{array}{l}\text { Turismo de base comunitária e cadeias produtivas; projeto de turismo e inclusão } \\
\text { social nos Parques, que trabalhe o público "quase-visitante", segmentos do turismo } \\
\text { que poderiam ser desenvolvidos (pesca esportiva, corridas de aventura), análise de } \\
\text { demanda turística; potencializar o turismo de base comunitária }\end{array}$ \\
\hline Caracterização & $\begin{array}{l}\text { Experiência do visitante; perfil de visitantes (percepções e motivações); Como usar } \\
\text { os dados de perfil, percepções e motivações na gestão da visitação? } \\
\text { Identificar/entender porque certos grupos não visitam os parques; Benefícios; } \\
\text { Entender melhor a dinâmica da visitação; Como mediar os conflitos entre visitantes } \\
\text { e proprietários do entorno da UC; Estudo de opinião do visitante com relação a } \\
\text { gestão das UC }\end{array}$ \\
\hline Impactos & $\begin{array}{l}\text { Impactos da visitação na fauna; Capacidade de suporte; Monitoramento da } \\
\text { visitação; Outras atividades de uso público que hoje não são permitidas ou não são } \\
\text { desenvolvidas: elas causam efetivamente impactos em todos os contextos? } \\
\text { (presença de cachorros, trilhas de mountain bike, turismo equestre, uso do fogo } \\
\text { [fogueira e churrasco], esportes aquáticos, interação com animais, camping, } \\
\text { eventos, corridas de aventura; Tecnologias para implementação e monitoramento }\end{array}$ \\
\hline
\end{tabular}




\begin{tabular}{|c|c|}
\hline & $\begin{array}{l}\text { da visitação. Monitoramento de impactos utilizando o Roteiro para Monitoramento } \\
\text { de Impactos do ICMBio; Pesquisa sobre o uso direto dos recursos. Ex.: Pesca } \\
\text { esportiva. Quais são efetivamente os impactos causados? Impactos ecológicos e } \\
\text { econômicos que trilhas de longo percurso podem gerar como corredores } \\
\text { ecológicos; Como lidar com o aumento da visitação; Analisar os impactos dos } \\
\text { contratos de concessão de serviços e sua repercussão no que diz respeito aos } \\
\text { objetivos de conservação da área; Avaliação de impactos da visitação em trilhas; } \\
\text { Qual o impacto positivo da visitação sobre a proteção das UC? }\end{array}$ \\
\hline Manejo & $\begin{array}{l}\text { Planos de manejo; zoneamento e sua relação com a visitação (são compatíveis e } \\
\text { atuais?); relação entre visitação e objetivo das categorias; estudo de outras } \\
\text { categorias, Possibilidade de visitação noturna; Gestão da visitação nos Caminhos } \\
\text { da Serra do Mar; Entender as demandas e a dinâmica de atuação dos Condutores de } \\
\text { Visitantes e dos Clubes Excursionistas; NBV de trilhas e atrativos; ordenamento de } \\
\text { campings em UC; Avaliação de estrutura; Identificação e envolvimento das partes } \\
\text { (unidades de conservação particulares e unidades de conservação municipais e } \\
\text { outras categorias de UC, inclusive REBIO); necessidade da zona intangível; } \\
\text { classificação de trilhas; trilhas de longo curso; infraestrutura de saneamento } \\
\text { aplicadas a campings e populações tradicionais e residentes em UC; Ordenamento } \\
\text { de atividades religiosas; ordenamento do turismo voltado para atividades marinhas. }\end{array}$ \\
\hline Sugestões & $\begin{array}{l}\text { Levar pesquisas para a Amazônia e o Nordeste, que têm outra dinâmica de } \\
\text { visitação e outras características; Ter uma plataforma que contenha todas as } \\
\text { iniciativas que estão sendo úteis; Como inserir os dados/resultados de pesquisa na } \\
\text { gestão }\end{array}$ \\
\hline
\end{tabular}

Fonte: Relatório do III Encontro da CPVAP, 2017.

No IV Encontro, para os questionamentos relacionados aos principais objetivos e temas da CPVAP foi utilizada a metodologia PAD, cujas palavras-chave foram agrupadas nos Quadros 3 e 4 .

QUADRO 3 - Principais palavras-chave relacionadas aos questionamentos apresentados no IV Encontro da CPVAP.

\begin{tabular}{|l|l|}
\hline Perguntas & \multicolumn{1}{|c|}{ Palavras Chave } \\
\hline "Por que"? & $\begin{array}{l}\text { Grupo forte; pesquisa qualificada; ambiente colaborativo; estímulo às parcerias; } \\
\text { geração de conhecimentos; gestão da visitação; cooperação; Reconexão das pessoas } \\
\text { com a natureza. }\end{array}$ \\
\hline "Para que" & $\begin{array}{l}\text { Integração do conhecimento; geração de procedimentos; geração de metodologias, } \\
\text { pessoas capacitadas instrumentos de pesquisa e protocolos; TBC; desenvolvimento } \\
\text { socioeconômico de comunidades; qualidade de vida, saúde pública e experiência do } \\
\text { visitante; conservação ambiental; compreensão da sociedade sobre o valor das APs; } \\
\text { Influenciar Políticas Públicas. }\end{array}$ \\
\hline $\begin{array}{l}\text { "Para } \\
\text { quem"? }\end{array}$ & $\begin{array}{l}\text { Gestores e funcionários das AP; universidades; pesquisadores; estudantes; operadores } \\
\text { de turismo; comunidade, empreendedores, Instituições de saúde; Meio Ambiente e }\end{array}$ \\
\hline
\end{tabular}

Fonte: Relatório do IV Encontro da CPVAP, 2018.

Ao considerar ainda as perguntas do método PAD, houve a identificação de diferentes núcleos dos sentidos para as perguntas "como?", "quem?" e "quando?" (Quadro 4), com a 
identificação de ações para atingir os diferentes objetivos. As palavras-chave apontaram para a necessidade de:

- Geração de procedimentos, metodologias e instrumentos de pesquisa;

- Aumento do número de pessoas capacitadas em pesquisa e manejo da visitação em UC;

- Promoção do turismo de base comunitária e o desenvolvimento socioeconômico de comunidades;

- Identificação de iniciativas que possam contribuir para a saúde e qualidade de vida por meio da visitação.

QUADRO 4 - Principais ações relacionadas aos questionamentos do IV ECPVAP.

\begin{tabular}{|l|l|}
\hline Como? Quem? e Quando? & \multicolumn{1}{|c|}{ Ações } \\
\hline $\begin{array}{l}\text { Gerar de procedimentos, } \\
\text { metodologias e instrumentos de } \\
\text { pesquisa }\end{array}$ & $\begin{array}{l}\text { Questionário perfil de Visitantes - 3 meses; Manual de aplicação } \\
\text { do questionário - 6 meses; pré-testes - 6 meses; Levantamento } \\
\text { preliminar - 6 meses. }\end{array}$ \\
\hline $\begin{array}{l}\text { Aumentar do número de } \\
\text { pessoas capacitadas em } \\
\text { pesquisa e manejo do uso } \\
\text { público em UC }\end{array}$ & $\begin{array}{l}\text { Criar Google Groups; Artigo sobre o "estado da arte"; Divulgar } \\
\text { o livro "Tourism in Protected Areas of Brazil"; Conferência } \\
\text { Internacional de Interpretação (capacitar em side events e cursos } \\
\text { de curta duração); Disciplinas (Gestores e professores); } \\
\text { Diagnóstico dos estudos e grupos de pesquisa; Parcerias para } \\
\text { curso de especialização em planejamento e gestão do turismo de } \\
\text { natureza; apoio financeiro; Desenvolver material de divulgação } \\
\text { da comunidade; Incentivar a participação dos membros da } \\
\text { comunidade de prática no TAPAS Group / IUCN. }\end{array}$ \\
\hline $\begin{array}{l}\text { Favorecer o turismo de base } \\
\text { comunitária e o } \\
\text { desenvolvimento } \\
\text { socioeconômico de } \\
\text { comunidades }\end{array}$ & $\begin{array}{l}\text { Demandar pesquisas; valorizar relato de experiências; Mapear e } \\
\text { caracterizar as iniciativas e demandas de pesquisa; Universidade } \\
\text { atuando como facilitadora dos arranjos institucionais; Colocar a } \\
\text { comunidade de prática em contato com o GT de TBC do } \\
\text { ICMBio. }\end{array}$ \\
\hline $\begin{array}{l}\text { Promover iniciativas para } \\
\text { contribuição da visitação na } \\
\text { saúde e qualidade de vida }\end{array}$ & $\begin{array}{l}\text { Realizar levantamento; Adoção de ferramentas de marketing } \\
\text { eficientes; "apelar ao bem-estar", da saúde e qualidade de vida } \\
\text { das comunidades locais; Melhorar e ampliar o sistema de trilhas; } \\
\text { Evitar a criação de meios interpretativos com o uso de mídias } \\
\text { digitais; Promover a adoção de alimentação saudável; Promover } \\
\text { o tratamento de resíduos. }\end{array}$ \\
\hline
\end{tabular}

Fonte: Relatório do IV ECPVAP, 2018.

Considerando o perfil dos participantes por encontro, pode-se afirmar que o III Encontro apresentou o maior número de participantes (Figura 1). Esse fato pode ser explicado pela participação de representantes de todas as universidades públicas do estado do Rio de Janeiro. 
FIGURA 1 - Gráfico com o número de participantes dos Encontros da CPVAP (Geral) distribuídos conforme o perfil e instituição de origem.

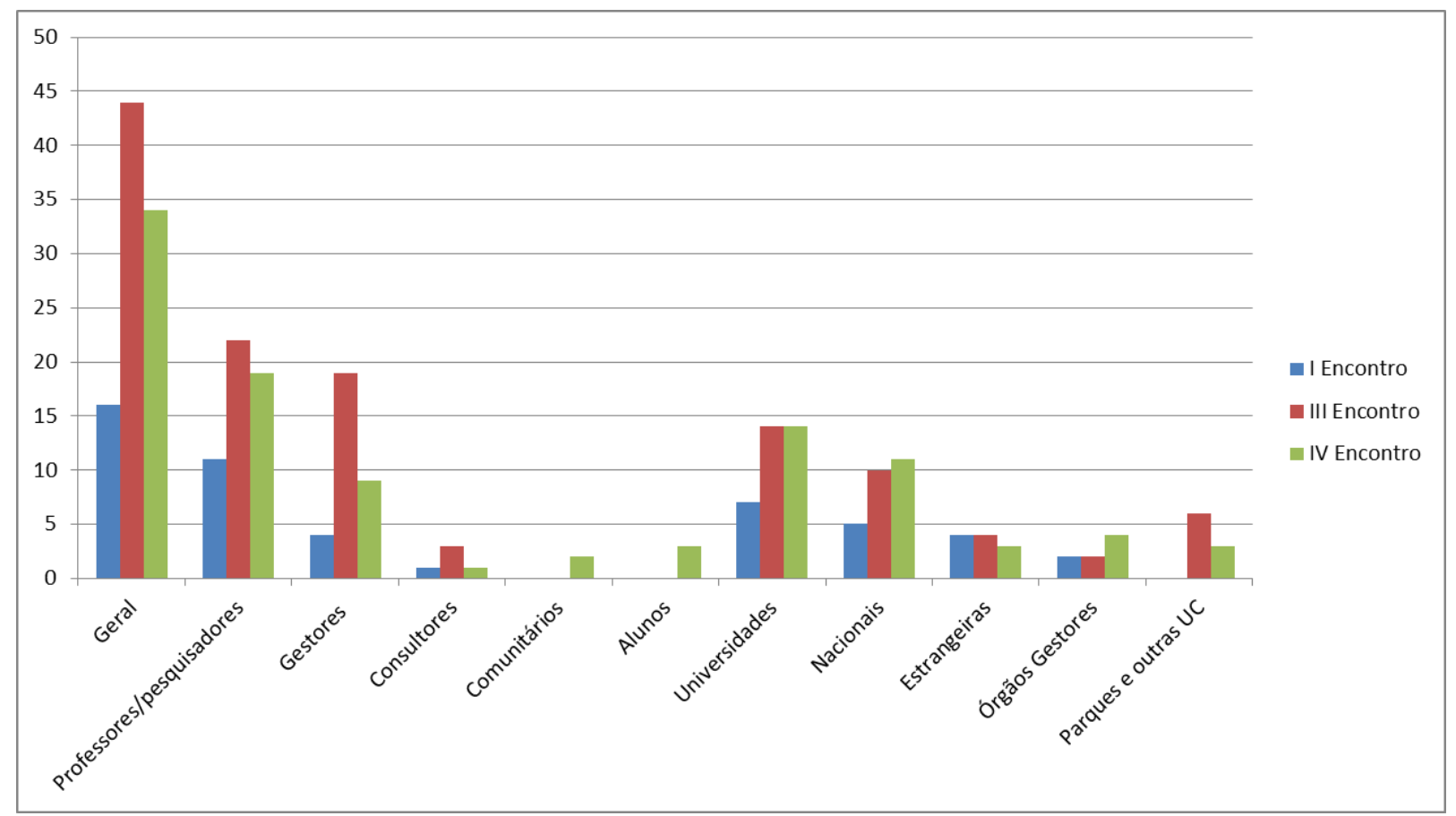

De acordo com a Figura 1, observa-se que o perfil da comunidade é formado por professores, pesquisadores e gestores, o que coaduna com os objetivos propostos para esta comunidade de prática, como proposto por Souza-Silva e Davel (2007). No entanto, a participação de maior diversidade de perfis pode enriquecer os debates, tais como alunos bolsistas (tanto da graduação quanto da pós-graduação), membros de grupos de populações locais e representantes de organizações não governamentais e outros órgãos de gestão. Dessa maneira ratifica-se o foco no processo de ensino-aprendizagem relatado em Ipiranga, Faria e Amorim (2008).

No I Encontro, o número de participantes foi inferior aos demais eventos pelo fato de que a Comunidade de Prática estava no seu momento inicial de estruturação. Os pesquisadores e gestores ainda estavam conhecendo o significado de uma Comunidade e seus objetivos e possibilidades de atuação.

Ao analisar a proporção de gestores e pesquisadores no evento, chega-se a conclusão que o III Encontro foi o mais equilibrado com relação a essa questão. Esse Encontro foi realizado na região sudeste do Brasil, e a participação de diferentes instituições foi motivada, sobretudo em função do acesso facilitado até o local de realização do evento, a sede Teresópolis do Parque Nacional Serra dos Órgãos. Este aspecto pode ser considerado como ponto forte da edição de 2017, e devido à qualidade agregadora do Encontro, o sentido de uma Comunidade de Prática foi melhor internalizado pelos participantes. 
Com relação ao IV Encontro, o número de participantes foi inferior ao evento de 2017, muito provavelmente pelo fato de ter sido realizado na região norte e de a maior parte dos membros residirem na região sudeste, o que dificultou a participação dos pesquisadores e gestores de UC já integrados a CPVAP, obrigando a um deslocamento mais dispendioso para a maioria. Por outro lado, o Encontro ocorrido em Manaus possibilitou maior diversidade de perfil, pois contou com a participação de comunitários envolvidos em iniciativas de turismo de base comunitária em Reservas Extrativistas, bem como de chefes de UC Federais da região e, também, de alunos de graduação e pós graduação. Esse fato indica que a CPVAP tem potencial para maior participação de representantes locais e trocas mais qualitativas quando os encontros ocorrem fora do eixo Rio - São Paulo - Brasília.

As palavras-chave relacionadas ao objetivo principal da CPVAP, destacadas pelos participantes nos relatórios dos três Encontros também foram analisadas. Foi considerado o total de 1.846 palavras, em um contexto geral de 32.083 palavras redigidas nas 184 páginas dos relatórios dos Encontros da Comunidade de Prática. Ainda considerando os objetivos, as palavras-chave mais citadas ao longo dos encontros da CPVAP estão relacionadas à pesquisa sobre a visitação em áreas protegidas (Figura 2).

FIGURA 2 - Gráfico com as palavras chave principais do objetivo da CPVA. Dados gerais dos três Encontros

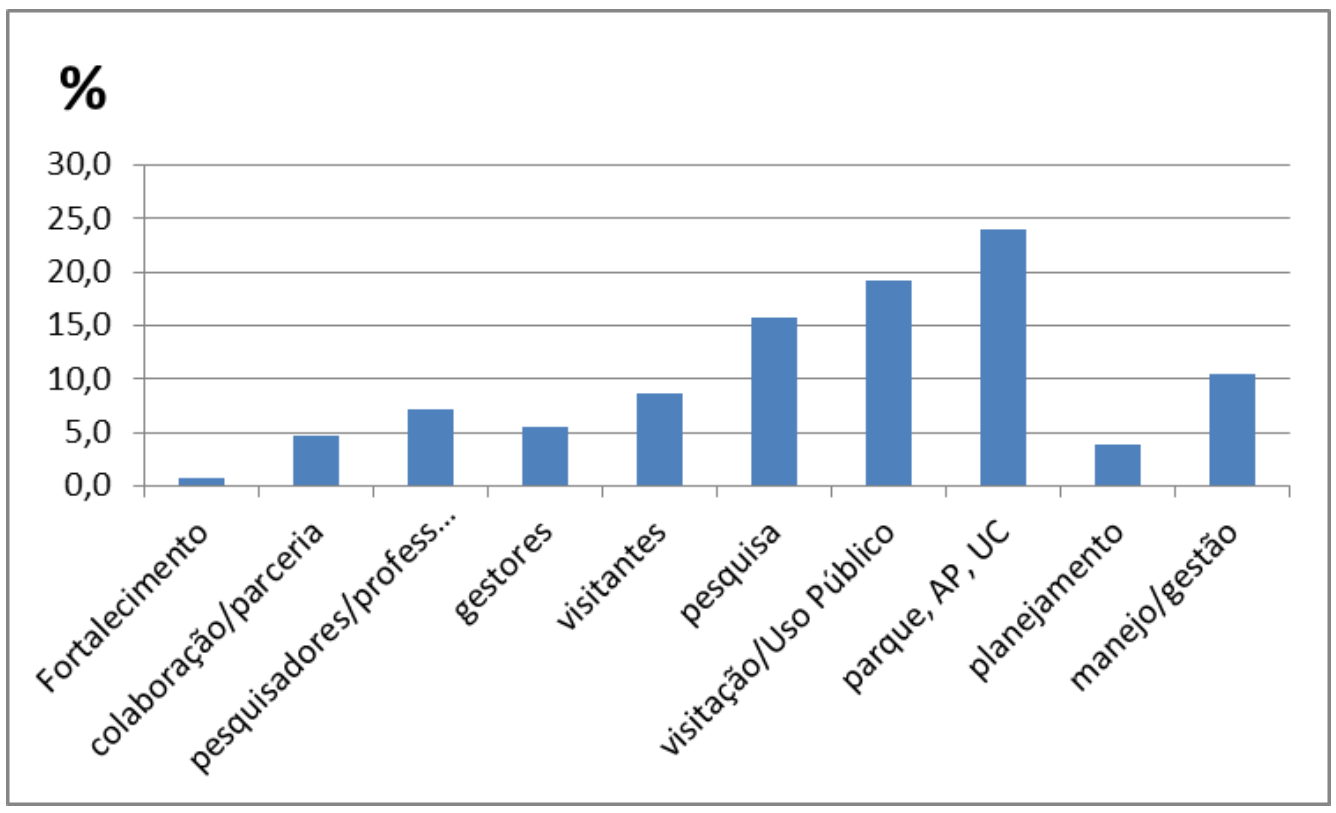

Nesse sentido, pode-se inferir que o setor acadêmico deseja ser demandado e ou apoiado no desenvolvimento de pesquisas que subsidiem a gestão da visitação em Unidades de Conservação. Interessante notar que essa relação não se deu uniformemente nos três encontros e isso pode ser explicado pelas diferentes metodologias para fomentar as discussões nos eventos (Figura 3). 
Revista Eletrônica Uso Público em Unidades de Conservação. Niterói, RJ. Vol. 7, no 11. 2019

\section{http://www.periodicos.uff.br/uso_publico}

FIGURA 3 - Gráfico com as palavras chave principais do objetivo da CPVA. Dados específicos por Encontro.

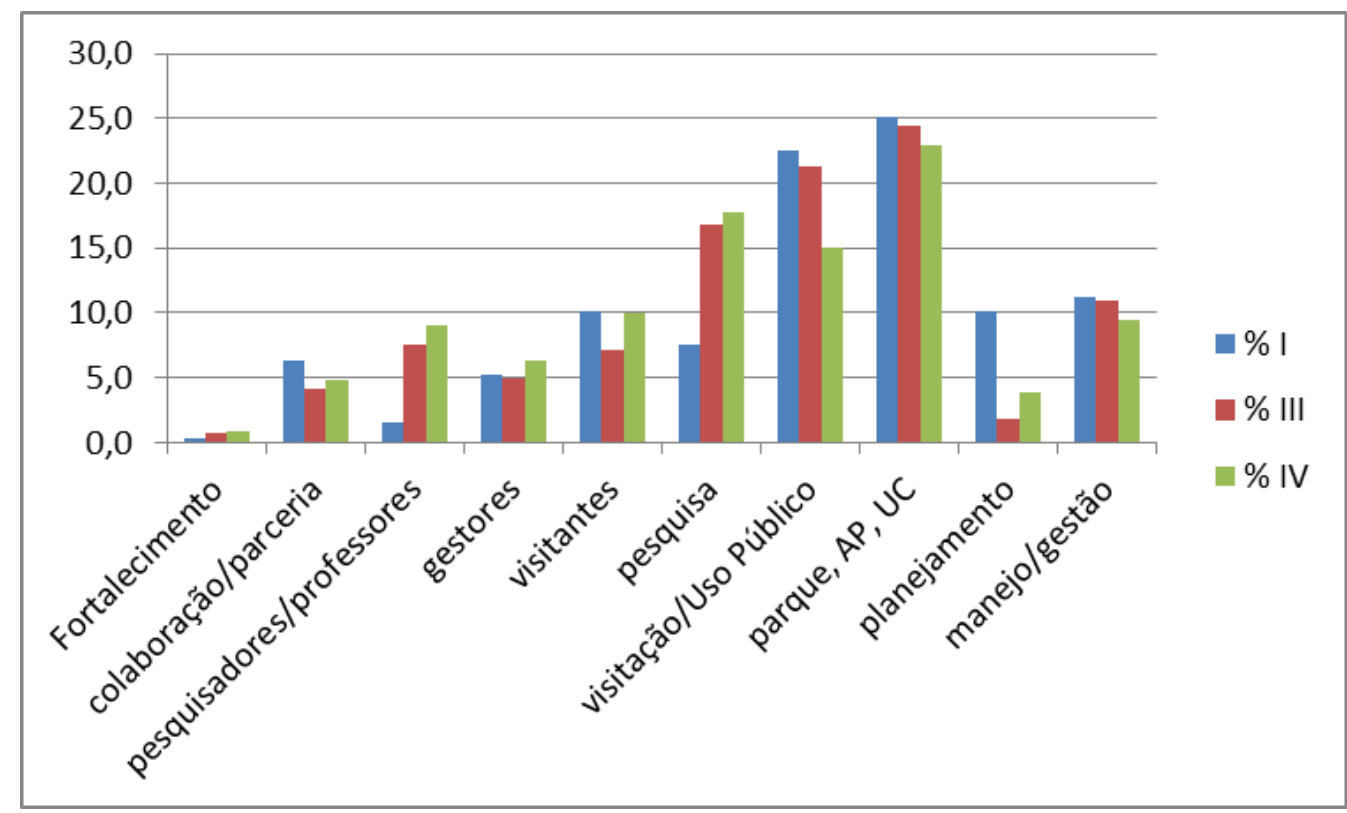

Ao longo de todos os encontros, os participantes elaboraram, ou fizeram de forma espontânea, perguntas para nortear os debates, totalizando 121 questões. As perguntas mencionadas versaram sobre diferentes temas. A Figura 4, a seguir, destaca as lacunas, o turismo e temas relacionados, o manejo de UC e a experiência dos visitantes de acordo com a frequência em que foram relatadas nos Encontros, a partir dos registros dos relatórios:

FIGURA 4. Gráfico com os sentidos dos questionamentos em todos os Encontros

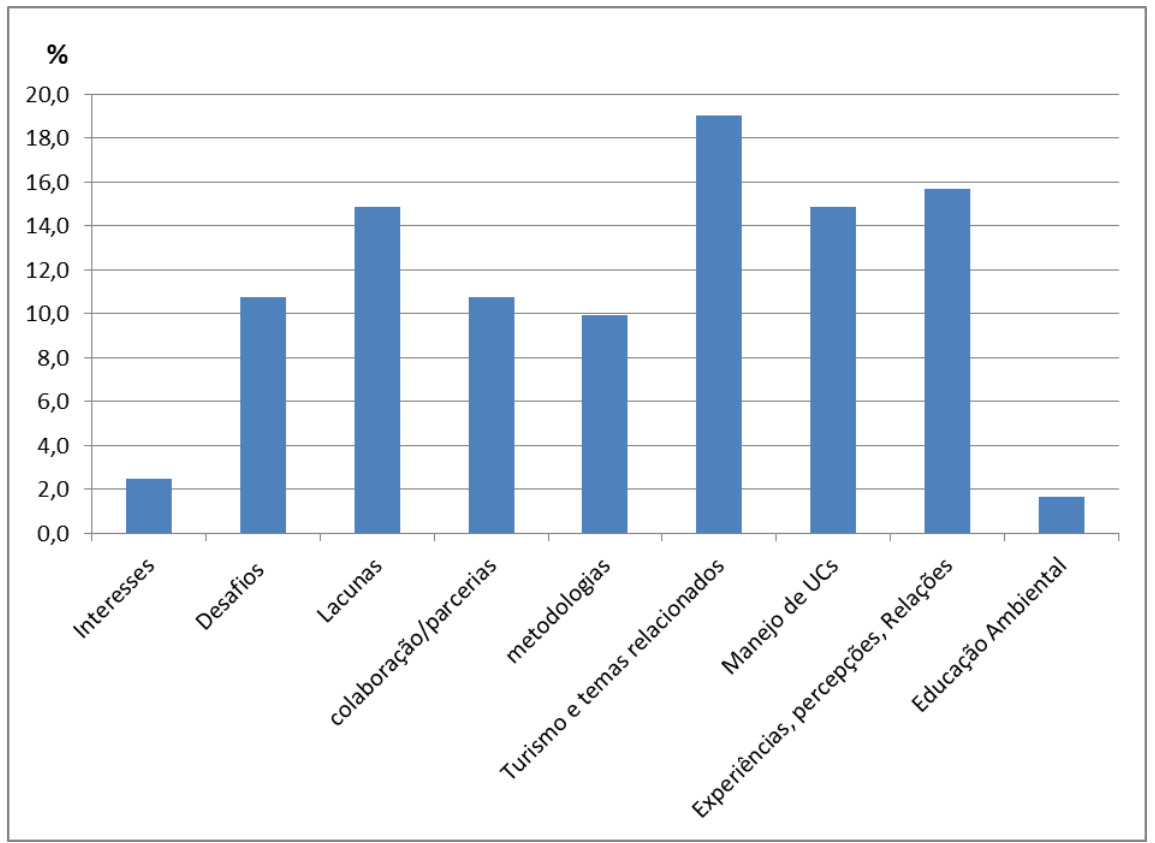


É interessante notar que, de maneira semelhante ao que aconteceu com as palavras-chave referentes aos objetivos da comunidade de prática, os temas dos questionamentos não se distribuíram de maneira uniforme entre os três encontros (Figura 5).

FIGURA 5: Gráfico com os percentuais de citação dos sentidos dos questionamentos, por encontro

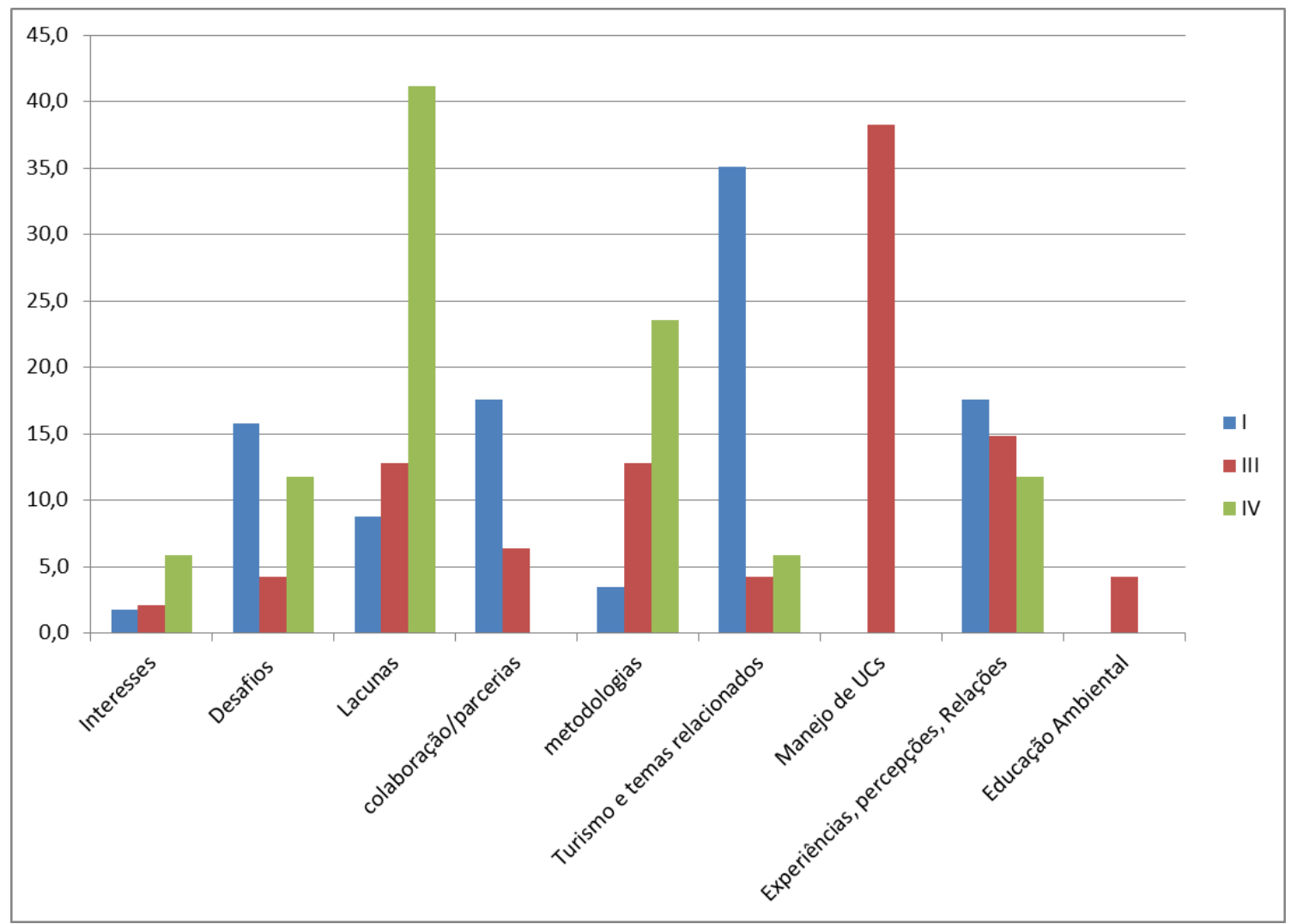

Pode-se observar que preocupações concernentes ao turismo em áreas protegidas prevaleceram no primeiro Encontro, enquanto que o Manejo de UC parece ter sido a preocupação central da terceira edição. $\mathrm{O}$ apontamento de lacunas foi o destaque no quarto Encontro (Figura 5). Dessa maneira, como destacado por Ipiranga, Faria e Amorim (2008) esse caráter fluido é um dos motivadores da inovação e aprendizado.

\section{CONSIDERAÇÕES FINAIS}

Dentre os desafios elencados no I Encontro da CPVAP destacam-se aqueles relacionados a necessidade de melhorar a conexão, comunicação e relacionamento com os visitantes e com as comunidades. $\mathrm{O}$ aprimoramento das relações entre pesquisadores e administradores de UC, em prol de um planejamento e gestão da visitação mais eficiente, balizada por pesquisas e consubstanciada nas experiências práticas também é elencado. Fica como aprendizado a necessidade de uma priorização dos inúmeros desafios e a síntese proporcionada pelo presente artigo pode contribuir para tal. 
No que diz respeito às linhas de atuação da Comunidade, o "manejo" foi a que apresentou uma maior diversidade de tópicos que devem ser trabalhados. Ficou evidente pela análise do conteúdo dos relatórios que os objetivos e a função da comunidade estão bem definidos. Contudo, os membros do grupo continuam refletindo e buscando caminhos para superar alguns desafios para colocar em prática o planejamento das ações.

Outro ponto que merece destaque refere-se a uma maior proporção de participantes da região Sudeste, indicando a necessidade de se ampliar a participação de pesquisadores e gestores das diferentes regiões brasileiras. A Comunidade de Prática de Visitação em Áreas Protegidas é uma iniciativa recente que ainda está na quinta edição, dessa forma espera-se que essas lacunas "geográficas" sejam superadas.

Constatou-se que o número de gestores de unidades de conservação aumentou entre o I e III encontros, mas depois diminuiu, talvez pelo fato de que o deslocamento até Manaus fosse mais oneroso ou pela mudança paulatina que vem ocorrendo junto aos Centros Especializados responsáveis pelo fomento e execução das pesquisas do ICMBio que vem procurando investir em pesquisa aplicada voltada para as demandas emergentes da própria instituição.

Quando houve maior presença desses gestores de unidades de conservação, houve o foco em questões sobre o manejo. Por fim, destaca-se que dentre os temas elencados para atuação conjunta entre gestores e pesquisadores, não houve aprofundamento sobre educação e interpretação ambiental.

A linha de atuação mais diversa identificada no II Encontro da CPVAP foi relacionada ao "manejo" e as maiores demandas foram relacionadas à avaliação e manejo de impactos da visitação.

No IV ECPVAP, o “para que?" apresentou a maior diversidade de sugestões aparentemente, os participantes dos encontros passaram a ter clareza dos objetivos da CPVAP. No entanto, ainda aparentam não se sabe "como" fazer. Somente um grupo chegou ao "como fazer" no IV Encontro da CPVAP.

Nesse sentido, apesar de haver a compreensão de que a academia pode e deve desenvolver pesquisas em UC, cresce a ideia em meio aos seus gestores de que a pesquisa acadêmica não acompanha ou não consegue "dar respostas" frente à complexidade, intensidade e a rapidez das questões que comprometem a atuação da gestão pública. Não é intenção explorar o tema nesse artigo, mas cabe mencionar que tal abordagem apareceu com certa frequência em meio aos debates travados no IV ECPVAP.

Diante dos resultados da análise, é importante apontar três observações imprescindíveis: 1) turismo, manejo e experiência dos visitantes são os termos mais citadas nos três relatórios; 2) quando houve maior presença de gestores de unidades de conservação, questões sobre o manejo dessas áreas protegidas foram o foco dos debates; 3) e não houve grande ênfase sobre interpretação e educação ambiental. 
Por fim, é importante ressaltar que a Comunidade de Prática de Visitação em Áreas Protegidas está discutindo sua estrutura de acordo com os objetivos propostos, e o número de questionamentos vem diminuindo a cada evento, o que pode indicar uma maior clareza das suas ações. De todo modo, o perfil e a dinâmica das relações entre os membros permitem afirmar que esta atende aos pressupostos de uma comunidade de prática, devido ao potencial ativado de troca de conhecimentos e experiências. Os membros da comunidade já reconhecem seus propósitos e possuem diretrizes definidas.

Destacam-se, como principais contribuições deste artigo a comunicação científica sobre a Comunidade de Prática de Visitação em Áreas Protegidas. A análise sobre processo de consolidação da comunidade de prática no tema em questão é importante, pois busca interpretar os resultados e indicar caminhos para os desafios encontrados, o que pode colaborar para a manutenção do ciclo virtuoso de estímulo a cooperação, atrair mais interessados ou inspirar novas iniciativas como esta.

\section{REFERÊNCIAS BIBLIOGRÁFICAS}

BARDIN, L. Análise de Conteúdo. Lisboa: Edições 70, 1977c. 229 p.

COLLEGE OF FOREST AND CONSERVATION. Brazil - US University Collaborative on Protected Area: Planning Meeting Report. Report I. 2015.

COMUNIDADE DE PRÁTICA DE VISITAÇÃO EM ÁREAS PROTEGIDAS. Encontro da Comunidade de Prática de Visitação em Áreas Protegidas, 3, Relatório III. 2017.

COMUNIDADE DE PRÁTICA DE VISITAÇÃO EM ÁREAS PROTEGIDAS. Encontro da Comunidade de Prática de Visitação em Áreas Protegidas, 4, Relatório IV. 2018.

FIEGE, K.; MUTISSE L. C.; FONTOURA, L.; QUIVE S. Configurar a Pesquisa em função da Prática: pesquisa Orientada para a Ação e Decisão (PAD). Berlim: Seminar für Ländliche Entwicklung (SLE); Humboldt-Universität zu Berlin, 2019. 190 p. Manual.

IPIRANGA, A. S. R.; FARIA, M. V. C. M.; AMORIM, M. A. A Comunidade de Prática da Rede NÓS: colaborando e compartilhando conhecimentos em arranjos produtivos locais. In: O\&S, v.15, n.44 - Janeiro/Março - 2008.

LAVE, J.; WENGER, E. Situated Learning: Legitimate Peripheral Participation. Cambridge: Cambridge University Press. 1991. http://dx.doi.org/10.1017/CBO9780511815355.

SOUZA-SILVA, J.; DAVEL, E. Da ação à colaboração reflexiva em comunidades de prática. Revista de administração de empresas, São Paulo, v.47, n.3, p.1-13, Sept. 2007. Available from <http://www.scielo.br/scielo.php?script=sci_arttext\&pid=S0034$75902007000300005 \& \operatorname{lng}=\mathrm{en} \& \mathrm{nrm}=\mathrm{iso}>$. access $\quad$ on $31 \quad$ Aug. 2019. http://dx.doi.org/10.1590/S0034-75902007000300005. 
Revista Eletrônica Uso Público em Unidades de Conservação. Niterói, RJ. Vol. 7, no 11. 2019 http://www.periodicos.uff.br/uso_publico

WENGER, E. Communities of practice: learning, meaning and identity. Cambridge: Cambridge University Press, 1998.

WENGER, E.; SNYDER, W. E. Communities of practice: the organizational frontier. In: Harvard business review. January - February. 2000. 